\title{
Endometriumkarzinom: Vorteil durch Sentinelbiopsie?
}

\begin{abstract}
Unter anderem beim Mammakarzinom hat sich das Sentinellymphknotenkonzept bewährt: Ist der Wächterlymphknoten frei von Metastasen, ist davon auszugehen, dass dies auch auf die nachgeschalteten Lymphknoten zutrifft. Daten der FIRES-Studie deuten darauf hin, dass dies für Patientinnen mit einem Endometriumkarzinom ebenfalls gilt.
\end{abstract}

$\mathrm{n}$ der prospektiven Studie FIRES haben Forscher Sensitivität und negativen prädiktiven Wert der Kartierung von Wächterlymphknoten (WLK; primärer Endpunkt) bei Patientinnen mit Endometriumkarzinom (EC) im klinischen Stadium 1 ermittelt. An der Studie nahmen 385 Patientinnen im medianen Alter von 63 Jahren und mit einem durchschnittlichen Body-Mass-Index von 33,4 $\mathrm{kg} / \mathrm{m}^{2}$ teil. In die Ektozervix wurde jeweils bei 3 und $9 \mathrm{Uhr}$ bis zu einer Tiefe von $1 \mathrm{~cm}$ der Farbstoff Indocyanin Grün injiziert. Nach der robotergestützten Kartierung mithilfe eines da-Vinci-Sioder-Xi-Systems erfolgte bei 340 Frauen eine komplette pelvine Lymphonodektomie (LNE). Nur bei etwas mehr als der Hälfte $(58 \%)$ wurden paraaortale Lymphknoten entfernt. Die paraaortale LNE wurde bei 74 der 100 Patientinnen vorgenommen, bei denen ein hochgradiger Tumor diagnostiziert worden war.

Insgesamt 41 Patientinnen (12\%) hatten ein metastasiertes EC, nachgewiesen anhand der WLK oder der durch LNE erhaltenen Proben. Bei 84\% der 340 Patientinnen entfernten die Chirurgen mindestens 10 Lymphknoten. Jeweils mindestens einen positiven WLK identifizierten sie bei $86 \%$. Insgesamt 888 WLK wurden mithilfe der Färbemethode lokalisiert. 36 der 293 Patientinnen mit mindestens einem positiven WLK hatten Metastasen in weiteren Lymphknoten, 35 (97\%) von ihnen wurden anhand der WLK korrekt diagnostiziert.

Es ergab sich eine Sensitivität für die Sentinellymphknotenbiopsie beim Nachweis einer Lymphknotenmetastasierung von $97,2 \%$ (95\%-Konfidenzintervall [95\%-KI] 85-100\%). Bei 257 der 258 Frauen mit negativen WLK waren alle anderen Lymphknotenbiopsien ebenfalls negativ. Der negative prädiktive Wert liegt demnach bei 99,6\% (95\%-KI 97,9$100 \%)$.

Fazit: Metastasen des Endometriumkarzinoms lassen sich mithilfe der Wächterlymphknotenbiopsie offenbar mit hoher Genauigkeit vorhersagen. Beim Staging des Karzinoms könnte sie den US-Ärzten zufolge den betroffenen Frauen eine LNE ersparen.

Peter Leiner

Rossi EC et al. A comparison of sentinel lymph node biopsy to lymphadenectomy for endometrial cancer staging (FIRES trial): a multicentre, prospective, cohort study. Lancet Oncol. 2017;18(3):384-92.

\section{Kommentar:}

Man mag kaum glauben, dass dieses prima vista hervorragende Ergebnis von Operateuren erzielt wurde, von denen $90 \% \mathrm{zu}$ Beginn der Studie keine Erfahrung mit Sentinellymphknotenbiopsien hatten.
Auch auf den zweiten Blick bleibt eine Reihe von Vorbehalten:

_Die "kompletten" LNE erfolgten nach US-amerikanischem Standard, d.h. in der Regel nur bis zur Arteria mesenterica inferior.

_ Es wurden im Mittel nur 19 Lymphknoten entfernt.

_ $72 \%$ der Patientinnen hatten ein EC mit niedrigem Rückfallrisiko (Low-Risk-EC).

_ Die Patientinnen mit hohem Rückfallrisiko (High-Risk-EC), die ja am häufigsten Lymphknotenmetastasen, insbesondere in der hohen Paraaortalregion haben, sind selbst nach Ansicht der Forscher in der Studie ungenügend berücksichtigt.

_ Bei $14 \%$ der Patientinnen $(n=47)$ konnte kein WLK dargestellt werden. Hiervon hatten 5 Frauen in der kompletten LNE Lymphknotenmetastasen.

\section{"Es bleibt eine Reihe von vorbehalten."}

_ In $40 \%$ der Fälle hätte bei nur einseitigem Nachweis eines WLK eine halbseitige komplette LNE durchgeführt werden müssen.

Wir wissen, dass Patientinnen mit einem Low-Risk-EC nicht von einer LNE profitieren. Da bei Low-Risk-EC die LNE keinen Nutzen hat, ist hier auch die Sentinellymphknotenbiopsie überflüssig.

Bei den High-Risk-EC wissen wir noch nicht, ob eine wie auch immer geartete LNE das Überleben der Patientinnen verbessert. Für diese Frauen ist die FIRES-Studie selbst nach Ansicht von Emma C. Rossi und Kollegen unzureichend.

Zurzeit laufen große randomisierte Studien, die den Nutzen eines diagnostischen Lymphknotensamplings (STATEC-Studie) bzw. einer systematischen therapeutischen Lymphonodektomie (ECLAT-Studie) klären sollen (Kasten 1). Wenn die STATEC- bzw. ECLAT-Studien zeigen sollten, dass eine LNE bei diesen Frauen keinen Überlebensvorteil bringt, wäre eine Sentinellymphknotenbiopsie ebenfalls überflüssig.

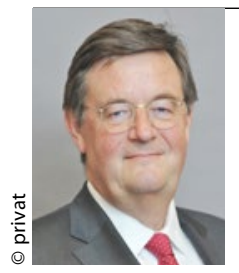

Prof. Dr. med. Günter Emons Direktor der UniversitätsFrauenklinik, Göttingen emons@med.unigoettingen.de 